DOI: http://dx.doi.org/10.18569/tempus.v14i1.2630

\title{
Gasto federal com ações de saúde bucal no Sistema Único de Saúde no período de 1995 a 2013
}

\section{Federal spending on public oral healthcare in Brazilian health system from 1995 to 2013}

\author{
Cínthia Sampaio Cristo ${ }^{1}$ \\ Áquilas Nogueira Mendes² \\ Paulo Frazão ${ }^{3}$ \\ Paulo Capel Narvai ${ }^{4}$
}

RESUMO: Uma inflexão significativa marcou, em 2004, a Política Nacional de Saúde Bucal, que passou a contar com mais recursos do governo federal. Neste estudo, de avaliação econômica em saúde, são analisadas as características dos aportes financeiros a essa política no período de 1995 a 2013, com vistas a identificar aproximações e afastamentos entre os diferentes governos. Foram utilizados dados secundários e o desenvolvimento e aplicação de um método para recuperar, processar e apurar as frações relativas aos gastos específicos com saúde bucal, tornou viável a análise. Os resultados elucidam dados conflitantes na literatura sobre o dimensionamento desses recursos. Constatou-se que houve relativa estabilidade no gasto público federal entre 1995 e 2002, cuja média anual foi de R\$ 689,9 milhões e aumento entre 2003 e 2013, com o volume médio de recursos alcançando $\mathrm{R} \$ 1,05$ bilhão. A diferença entre os aportes correspondeu a um aumento de 51,5\%. A fração odontológica no gasto total com saúde no orçamento do Ministério da Saúde passou de $1,22 \%$ no período de 1995 a 2002 para $1,49 \%$ entre 2003 e 2013, um aumento de 0,27 pontos percentuais ou $22 \%$ da fatia inicial.

DESCRITORES: Política Pública. Saúde Bucal. Assistência Odontológica. Financiamento da Assistência à Saúde. Sistema Único de Saúde.

ABSTRACT: A significant inflection in 2004 marked the National Oral Health Policy, which now has more resources from the federal government. In this study of economic evaluation in health, the characteristics of the financial contributions to this policy in the period from 1995 to 2013 was analyzed, for the purpose of identifying similarity and differences between the different governments. Secondary data were used, and the development and application of a method to recover, process

1Departamento de Política, Gestão e Saúde da FSP/USP.

2 Professor Associado do Departamento de Política, Gestão e Saúde da FSP/USP.

3 Professor titular do Departamento de Política, Gestão e Saúde da FSP/USP.

4 Departamento de Política, Gestão e Saúde da FSP/USP.

ISSN 1982-8829 Tempus, actas de saúde colet, Brasília, 14(1), 103-114 mar, 2020. Epub Mai/2020 
and calculate fractions related to specific oral health expenditures made the analysis viable. The results elucidate conflicting data in the literature on the amount of these resources. It was found that there was relative stability in federal public spending between 1995 and 2002, whose annual average was R\$ 689.9 million and increase between 2003 and 2013, with the average volume of resources reaching $\mathrm{R} \$ 1.05$ billion. The difference between the contributions corresponded to an increase of $51.5 \%$. The dental care fraction of total health expenditure in the Ministry of Health budget increased from $1.22 \%$ in the period from 1995 to 2002 , to $1.49 \%$ between 2003 and 2013, an increase of 0.27 percentage points or $22 \%$ of the initial share.

DESCRIPTORS: Public Policy. Oral Health. Dental Care. Health Care Financing. Unified Health System.

RESUMEN: Una inflexión significativa marcó, en 2004, la Política Nacional de Salud Oral, que ahora tiene más recursos del gobierno federal. En este estudio, del ámbito de la economía de la salud, se analizan las características de las contribuciones financieras a esta política de 1995 a 2013, con el fin de identificar apoyos y desinterés desde los diferentes gobiernos. Se utilizaron datos secundarios y el desarrollo y la aplicación de un método para recuperar, procesar y calcular fracciones relacionadas con gastos específicos de salud bucal, hicieron posible el análisis. Los resultados aclaran datos contradictorios en la literatura sobre el tamaño de estos recursos. Se encontró que hubo una relativa estabilidad en el gasto público federal entre 1995 y 2002, cuyo promedio anual fue de $\mathrm{R} \$ 689,9$ millones y aumentó entre 2003 y 2013, con un volumen promedio de recursos que alcanzó R \$1,05 mil millones. La diferencia entre las contribuciones correspondió a un aumento del 51,5\%. La fracción dental en el gasto total en salud en el presupuesto del Ministerio de Salud pasó del 1,22\% de 1995 a 2002 al 1,49\% entre 2003 y 2013, un aumento de 0,27 puntos porcentuales o $22 \%$ del segmento inicial.

DESCRITORES: Política pública. Salud Publica Oral. Cuidado Dental. Financiamiento de la atención a la salud. Sistema Unico de Salud.

\section{INTRODUÇÃO}

Com a realização da $3^{\text {a }}$ Conferência Nacional de Saúde Bucal ( $\left.3^{\mathrm{a} C N S B}\right)$ foi concluído, em 2004, o processo político que produziu uma inflexão significativa no rumo dessa política pública setorial no âmbito do Sistema Único de Saúde (SUS). A $3^{\text {a }}$ CNSB aprovou 298 propostas para a Saúde Bucal direcionadas ao Estado e à sociedade. Não houve, nesse conjunto, uma proposta sequer cujo conteúdo colidia com alguma orientação do documento "Diretrizes da Política Nacional de Saúde Bucal" que o Ministério da Saúde encaminhara ao Conselho Nacional de Saúde (CNS), Conselho Nacional de Secretários Estaduais de Saúde (CONASS) e Conselho Nacional de Secretarias Municipais de Saúde (CONASEMS) e que, aprovado no âmbito desses conselhos, começara a ser implementado em todo o País ${ }^{(1)}$.

Incluídas no "Plano Nacional de Saúde: um pacto pela saúde no Brasil”, objeto da Portaria MSTempus, actas de saúde colet, Brasília, 14(1), 103-114 mar, 2020. Epub Mai/2020 ISSN 1982-8829 
GM 2.607, de 10/12/2004(2), as referidas Diretrizes consubstanciaram o que viria a ser conhecido como "Programa Brasil Sorridente" (PBS), cuja implementação teve abrangência efetivamente nacional, com maior ou menor ênfase, dependendo das relações políticas entre os entes federativos dos três níveis de governo ${ }^{(3)}$.

Transformado em uma das principais marcas simbólicas dos governos de Luiz Inácio Lula da Silva (2003-2010) na área da saúde, ao lado do 'SAMU' e do 'Farmácia Popular', o PBS passou a contar com um volume inédito de recursos, o que tornou factíveis iniciativas que viriam a se confundir com o próprio PBS, como os Centros de Especialidades Odontológicas (CEO) e os Laboratórios Regionais de Prótese Dentária (LRPD), dentre outras, como as Equipes de Saúde Bucal (ESB) na Estratégia Saúde da Família. Embora as ESB tenham sido iniciativa do governo de Fernando Henrique Cardoso, os CEO e os LRPD foram instituídos como parte do PBS e se constituíram em contribuições originais do governo Lula à Política Nacional de Saúde Bucal (PNSB) que, para isto, ampliou os recursos alocados a esta política ${ }^{(4)}$, a qual teve prosseguimento no governo que se seguiu, sob a Presidência de Dilma Rousseff. Na literatura científica brasileira que se ocupa do financiamento da PNSB e, mais especificamente, do PBS, há menções a ampliações dos recursos destinados a essa área ${ }^{(5)}$, que teriam alcançado cifras expressivas, chegando a 944,2\%, no período entre 2002 e $2009^{(6)}$. Kornis et al (2011) ponderam, porém, que o financiamento da saúde bucal através de incentivos federais teve início em 2000, no âmbito da atenção básica, com a inserção das ESB no então Programa Saúde da Família, e na atenção secundária em 2004, com os repasses financeiros para a implantação e custeio dos CEO e dos LRPD, processo este que teria se caracterizado por um "aumento contínuo e crescente de recursos financeiros", atravessando "dois governos de orientação política e ideológica, a princípio, bastante distintas"(7).

No processo de avaliação de políticas públicas há amplo reconhecimento sobre a necessidade de averiguar as características do financiamento de uma determinada política pública, para saber o quão é efetivamente implementada pelo gestor público, para além do que afirma a publicidade oficial. Arretche (2003) assinala que o processo de construção do desenho institucional de uma política nunca acaba, pois requer um contínuo aperfeiçoamento e porque todo desenho de política é resultado do conflito entre distintos grupos de interesse ${ }^{(8)}$. Decorre dessa assunção a importância de, à luz da avaliação econômica em saúde, analisar o grau dos investimentos realizados em determinadas políticas públicas, em diferentes contextos históricos, para empreender comparações e formar juízos de valor, indispensáveis ao processo de avaliação em saúde ${ }^{(9)}$.

Neste artigo o foco da análise é colocado, especificamente, sobre o período histórico de 1995 a 2013, de modo a caracterizar e dimensionar o padrão do gasto público federal em ações de saúde bucal no SUS, com vistas a identificar aproximações e afastamentos entre os referidos governos e, também, se houve efetivamente aumento contínuo e crescente dos recursos federais alocados à área.

ISSN 1982-8829 Tempus, actas de saúde colet, Brasília, 14(1), 103-114 mar, 2020. Epub Mai/2020 


\section{MÉTODO}

Trata-se de um estudo de avaliação econômica em saúde, conduzido a posteriori, com foco na dimensão da alocação de recursos financeiros a uma política pública setorial, em um contexto histórico bem definido, ou seja, o que abrangeu os governos de Fernando Henrique Cardoso (1995 a 2002), Luiz Inácio Lula da Silva (2003 a 2010) e parte do governo de Dilma Rousseff (2011 a 2013). Foram utilizados dados secundários obtidos junto ao Ministério da Saúde (MS).

O dimensionamento dos recursos alocados às ações ambulatoriais foi feito a partir de dados disponibilizados pelo Datasus. Tendo em vista as diferentes características que marcaram o processo de alocação de recursos no período considerado, foi necessário construir uma estratégia de busca e padronização dos valores, uma vez que os dados brutos não eram diretamente comparáveis entre si para todo o período abrangido por este estudo, sendo esta uma condição sine qua non para viabilizar o dimensionamento e a comparação proposta. Para esta finalidade, os dados foram buscados na página "Informações de Saúde" e, nela, o bloco de "Assistência à Saúde" e "Produção ambulatorial - de 1994 a 2007" e "Produção ambulatorial, por local de atendimento - a partir de 2008", definindo-se como área de abrangência a opção "Brasil por região e Unidades da Federação". Foi feita uma compatibilização e sistematização dos dados registrados no Sistema de Informações Ambulatoriais (SIA), tendo em vista as mudanças introduzidas nesse sistema e nas modalidades de repasse ao longo desse período. Por exemplo, a partir de 2006 os repasses do MS foram identificados em blocos de financiamento (atenção básica, média e alta complexidade, assistência farmacêutica, vigilância em saúde e gestão). No sítio eletrônico Portal da Transparência (http://aplicacao.saude.gov.br/portaltransparencia) foram obtidos, sistematizados e tabulados, especificamente para gastos com assistência odontológica, os dados relativos ao PAB Fixo, ao PAB Variável, ao Fundo de Ações Estratégicas e Compensação (FAEC), ao Limite Financeiro da Média e Alta Complexidade Ambulatorial e Hospitalar ("Teto MAC"), e ao Pacto pela Gestão do SUS. Todos os valores nominais apurados eram líquidos, uma vez que o interesse da análise recaiu sobre os recursos efetivamente repassados.

Tais opções metodológicas implicaram admitir as seguintes limitações: a) o foco na produção ambulatorial decorreu do fato de que, no caso da saúde bucal, no período do estudo, os procedimentos hospitalares eram raros e impactavam pouco no resultado final; b) a tabulação do PAB Fixo relativa a ações odontológicas foi possível criando-se uma "fração odontológica" (inexistente no PAB Fixo), composta a partir de 28 procedimentos odontológicos básicos nos anos de 1998, 1999 e 2000, quando estes ainda eram apresentados valorados na tabela SIA-SUS, cujo valor médio foi de $\mathrm{R} \$ 1,25$ por procedimento; c) a tabulação do PAB Variável relativa a ações odontológicas foi feita selecionando-se o item "Piso da Atenção Básica Variável" e considerando-se, ao final do processo de sucessivas seleções, apenas os valores relatados para dois componentes principais de interesse, denominados "Saúde Bucal" (que são os valores mais relevantes, em parcelas contínuas, relacionados à existência de equipes de saúde bucal na Estratégia de Saúde da Família) e "Incentivo Adicional Saúde Bucal" (que são repasses em parcelas únicas, relacionados com a 
aquisição de equipamentos), e acrescidos de outros três componentes com valores menores, criados a partir de 2011 ("Equipe de Saúde Bucal - Unidade Odontológica Móvel”, "Incentivo Adicional Unidade Odontológica Móvel”, “Ampliação da Resolutividade da Saúde Bucal”); d) a tabulação do FAEC relativa à saúde bucal foi processada a partir de dados obtidos para sete componentes, denominados: "Ortodontia" (com valores relatados de 2001 a 2008), "Prótese Dentária" (com valores relatados de 2005 a 2008), "OPM em Odontologia”(com valores relatados em 2008, 2009 e 2011), "Tratamentos Odontológicos" (com valores relatados de 2008 a 2010), "FAEC AIH Tratamentos Odontológicos" (com valores relatados de 2011 a 2013), "FAEC SIA - Tratamentos Odontológicos" (com valores relatados de 2011 a 2013) e "FAEC SIA - OPM em Odontologia" (com valores relatados de 2011 a 2013); e) a tabulação do "Teto MAC" relativa à saúde bucal foi processada a partir da seleção da opção componente denominado "Centro de Especialidades Odontológicas" (com valores relatados a partir de 2004, referentes ao custeio dos Centros de Especialidades Odontológicas); f) a tabulação dos valores do Pacto pela Gestão do SUS relativa à saúde bucal foi possível pela seleção do item "Implantação de Ações e Serviços de Saúde" especificando-se o componente denominado "Incentivo Adicional ao CEO" (com valores relatados de 2004 a 2009, referentes a repasses de incentivos destinados ao apoio na implantação de novos Centros de Especialidades Odontológicas).

Reitera-se que tais procedimentos metodológicos foram indispensáveis para obter os dados apurados para viabilizar a presente análise. Cabe registrar, ainda, que os valores apurados, em reais, provêm de um período histórico ainda marcado por inflação recorrente e por vários planos econômicos para controlá-la, sendo necessário, em decorrência, efetuar-se a correção dos valores monetários nominais, deflacionando-os. Esta operação constitui um imperativo metodológico, pois segundo MUNHOZ (1989) ${ }^{(10)}$, em um contexto inflacionário, a observação de uma série de valores monetários crescentes não permite concluir sobre o que realmente ocorreu, sem que se processe à deflação dos valores nominais. Neste estudo optou-se pelo Índice Geral de Preços Disponibilidade Interna (IGP-DI), uma vez que se trata de um índice composto pela ponderação de três outros índices que aferem tanto o custo de vida das famílias, o Índice de Preços ao Consumidor (IPC), quanto os setores bem delimitados da economia, representados pelo Índice de Preços no Atacado (IPA) e o Índice Nacional da Construção Civil (INCC). A abrangência geográfica destes três índices, o universo de variações de preços e a faixa de renda da população analisada (1 a 33 salários mínimos) faz do IGP-DI a medida mais aproximada da inflação nacional ${ }^{(11)}$. Para correção dos valores monetários os dados foram deflacionados utilizando-se o IGP/DI médio de cada ano, apurados pela Fundação Getúlio Vargas. Os valores obtidos foram convertidos em preços de dezembro de 2013 e foram calculadas estatísticas para dois períodos: 1995 a 2002 e de 2003 a 2013.

\section{RESULTADOS}

A Tab.1 mostra a evolução do gasto público federal sob a vigência da tabela SIA/SUS, nos anos 1995, 1996 e 1997. A Tab. 2 abrange os anos 1998, 1999 e 2000, período em que vigia a Norma Operacional Básica (NOB) 1996, incluindo, portanto, recursos provenientes das fontes 
$108 / /$

tabela SIA/SUS e PAB fixo. A Tab. 3 abrange o período de 2001 a 2005, sob influência das Normas Operacionais de Atenção à Saúde de 2001 e 2002 e do Pacto pela Gestão do SUS, computando-se recursos provenientes de várias fontes (tabela SIA/SUS, PAB Fixo e Variável e FAEC) e ainda, para os anos 2004 e 2005, as fontes "Teto MAC" e Gestão do SUS. A Tab. 4 reúne dados provenientes de várias fontes federais e abrange o período de 2006 a 2013. Esse conjunto de tabelas (Tab.1 a 4), apresenta a evolução do gasto federal com ações odontológicas ambulatoriais no período de 1995 a 2013, com os valores deflacionados (mês-base $=$ dez/2013), discriminados segundo cada componente de financiamento, de acordo com as diferentes normas que impactaram a gestão dos recursos financeiros da saúde. A Tab. 5 mostra a evolução do gasto público federal com saúde e com ações odontológicas ambulatoriais, no âmbito do MS, no período de 1995 a 2013.

Tabela 1 - Gasto público federal, em reais deflacionados, com ações odontológicas ambulatoriais de 1995 a 1997.

\begin{tabular}{lr}
\hline ANO & $\begin{array}{r}\text { TOTAL } \\
\text { (em R\$) }\end{array}$ \\
\hline 1995 & $718.742 .675,00$ \\
1996 & $660.740 .704,64$ \\
1997 & $635.768 .409,53$ \\
\hline
\end{tabular}

Valores deflacionados a preços de dez/2013 (IGP-DI/FGV)

Tabela 2 - Gasto público federal, em reais deflacionados, com ações odontológicas ambulatoriais de 1998 a 2000

\begin{tabular}{|c|c|c|c|}
\hline ANO & $\begin{array}{l}\text { SIA/SUS } \\
\text { (em R\$) }\end{array}$ & $\begin{array}{c}\text { PAB Fixo Odonto } \\
(\mathrm{em} \mathrm{R} \$)\end{array}$ & $\begin{array}{l}\text { TOTAL } \\
\text { (em R\$) }\end{array}$ \\
\hline 1998 & $77.304 .133,84$ & $712.059 .030,51$ & $789.363 .164,34$ \\
\hline 1999 & $74.426 .249,13$ & $648.183 .777,31$ & $722.610 .026,44$ \\
\hline 2000 & $56.312 .078,87$ & $590.057 .889,80$ & $646.369 .968,67$ \\
\hline
\end{tabular}


Tabela 3 - Gasto público federal, em reais deflacionados, com ações odontológicas ambulatoriais de 2001 a 2005

\begin{tabular}{|c|c|c|c|c|c|c|c|}
\hline ANO & $\begin{array}{l}\text { SIA/SUS } \\
\text { (em R\$) }\end{array}$ & $\begin{array}{l}\text { PAB Fixo } \\
\text { Odonto } \\
\text { (em RS) }\end{array}$ & $\begin{array}{c}\text { PAB Variável } \\
\text { Odonto } \\
(\mathrm{em} R S)\end{array}$ & $\begin{array}{c}\text { FAEC } \\
\text { Odonto } \\
(\mathrm{emRS})\end{array}$ & $\begin{array}{c}\text { Teto MAC } \\
\text { Odonto } \\
\text { (em RS) } \\
\end{array}$ & $\begin{array}{c}\text { Gestão do } \\
\text { SUS } \\
\text { (em RS) } \\
\end{array}$ & $\begin{array}{l}\text { TOTAL } \\
\text { (em R\$) }\end{array}$ \\
\hline 2001 & 62.632.994,63 & $542.806 .025,34$ & $48.118 .053,89$ & $853.647,49$ & - & - & $654.410 .721,35$ \\
\hline 2002 & $66.965 .005,59$ & $484.458 .409,00$ & $137.817 .765,12$ & $2.264 .073,63$ & - & - & $691.505 .253,33$ \\
\hline 2003 & $60.749 .634,55$ & $399.586 .333,62$ & $147.335 .495,58$ & $3.423 .642,23$ & - & - & $611.095 .105,98$ \\
\hline 2004 & $60.168 .439,83$ & $369.855 .134,03$ & $319.875 .346,30$ & $10.541 .773,50$ & $875.882,91$ & $5.038 .556,91$ & $766.355 .133,48$ \\
\hline 2005 & $60.785 .575,28$ & $358.925 .212,83$ & $437.721 .382,61$ & $13.566 .373,65$ & $22.746 .211,71$ & $25.536 .170,89$ & $919.280 .926,96$ \\
\hline
\end{tabular}

Valores deflacionados a preços de dez/2013 (IGP-DI/FGV)

Tabela 4 - Gasto público federal, em reais deflacionados, com ações odontológicas ambulatoriais, de 2006 a 2013

\begin{tabular}{|c|c|c|c|c|c|c|c|}
\hline ANO & $\begin{array}{l}\text { SIA/SUS } \\
\text { (Bloco } \\
\text { MAC) } \\
\text { (em R\$) }\end{array}$ & $\begin{array}{c}\text { PAB Fixo } \\
\text { Odonto } \\
\text { (Bloco AB) } \\
\text { (em R\$) }\end{array}$ & $\begin{array}{l}\text { PAB Variável } \\
\text { Odonto } \\
\text { (Bloco AB) } \\
\text { (em R\$) }\end{array}$ & $\begin{array}{l}\text { FAEC } \\
\text { Odonto } \\
\text { (Bloco } \\
\text { MAC) } \\
\text { (em RS) }\end{array}$ & $\begin{array}{c}\text { Teto MAC } \\
\text { Odonto } \\
\text { (Bloco MAC) } \\
\text { (em R\$) }\end{array}$ & $\begin{array}{c}\text { Gestão do } \\
\text { SUS } \\
\text { (Bloco } \\
\text { Gestão) } \\
\text { (em RS) }\end{array}$ & $\begin{array}{l}\text { TOTAL } \\
\text { (em R\$) }\end{array}$ \\
\hline 2006 & $62.778 .865,53$ & $357.793 .517,63$ & $558.337 .585,81$ & $14.215 .192,41$ & $61.032 .779,98$ & $19.999 .743,34$ & $1.074 .157 .684,71$ \\
\hline 2007 & $69.017 .046,81$ & $345.166 .125,83$ & $629.725 .742,46$ & $16.448 .205,86$ & $80.235 .169,15$ & $6.592 .122,17$ & 1.147.184.412,29 \\
\hline 2008 & $70.755 .041,42$ & $310.767 .515,80$ & $616.547 .143,58$ & $14.530 .732,00$ & $78.180 .413,93$ & $6.149 .371,95$ & $1.096 .930 .218,67$ \\
\hline 2009 & $74.401 .814,52$ & $308.306 .547,62$ & $654.779 .894,69$ & $692.449,84$ & $108.078 .342,22$ & $4.791 .702,94$ & $1.151 .050 .751,83$ \\
\hline 2010 & $73.732 .974,04$ & $290.912 .981,99$ & $723.859 .657,07$ & $109.176,05$ & $98.766 .674,96$ & 0,00 & 1.187.381.464,11 \\
\hline 2011 & $67.376 .924,79$ & $270.350 .202,10$ & $727.096 .598,10$ & $12.872,53$ & $83.170 .474,55$ & 0,00 & 1.148.007.072,06 \\
\hline 2012 & $61.968 .327,96$ & $257.178 .488,31$ & $826.466 .237,62$ & $9.013 .202,48$ & $115.327 .236,27$ & 0,00 & $1.269 .953 .492,64$ \\
\hline 2013 & $56.355 .191,73$ & $251.290 .892,50$ & $688.285 .130,00$ & $8.081 .798,42$ & $125.445 .650,00$ & 0,00 & $1.129 .458 .662,65$ \\
\hline
\end{tabular}

Valores deflacionados a preços de dez/2013 (IGP-DI/FGV)

Os municípios e estados que não implantaram Centro de Especialidades Odontológicas continuam recebendo os recursos financeiros federais a partir da série histórica informada no SIA, dentro do bloco MAC. 
Tabela 5 - Participação das ações odontológicas ambulatoriais no gasto público federal com saúde, em reais deflacionados, no período de 1995 a 2013

\begin{tabular}{|c|c|c|c|}
\hline ANO & $\begin{array}{c}\text { GASTO PÚBLICO FEDERAL COM SAÚDE } \\
\text { (Orçamento do Ministério da Saúde) } \\
\text { (em milhões de R\$) }\end{array}$ & $\begin{array}{c}\text { GASTO PÚBLICO FEDERAL } \\
\text { COM AÇÕES ODONTOLÓGICAS } \\
\text { AMBULATORIAIS } \\
(\mathrm{em} \text { milhões de RS) } \\
\end{array}$ & $\%$ \\
\hline 1995 & 55.197 & 719 & 1,30 \\
\hline 1996 & 50.478 & 661 & 1,31 \\
\hline 1997 & 58.303 & 636 & 1,09 \\
\hline 1998 & 55.327 & 789 & 1,43 \\
\hline 1999 & 59.833 & 723 & 1,21 \\
\hline 2000 & 58.319 & 646 & 1,11 \\
\hline 2001 & 58.353 & 654 & 1,12 \\
\hline 2002 & 56.588 & 691 & 1,22 \\
\hline 2003 & 50.635 & 611 & 1,21 \\
\hline 2004 & 55.683 & 766 & 1,38 \\
\hline 2005 & 58.638 & 919 & 1,57 \\
\hline 2006 & 64.365 & 1.074 & 1,67 \\
\hline 2007 & 66.592 & 1.147 & 1,72 \\
\hline 2008 & 65.777 & 1.097 & 1,67 \\
\hline 2009 & 77.365 & 1.151 & 1,49 \\
\hline 2010 & 77.924 & 1.187 & 1,52 \\
\hline 2011 & 83.819 & 1.148 & 1,37 \\
\hline 2012 & 85.521 & 1.270 & 1,49 \\
\hline 2013 & 85.607 & 1.129 & 1,32 \\
\hline PERÍODO & VALOR MÉDIO & VALOR MÉDIO & \\
\hline 1995-2002 & 56.550 & 690 & 1,22 \\
\hline 2003-2013 & 70.175 & 1.045 & 1,49 \\
\hline
\end{tabular}

Observa-se que houve relativa estabilidade no gasto público federal até 2002 e aumento entre 2003 e 2013, com o volume de recursos evoluindo de aproximadamente R\$ 689,9 milhões em média no período de 1995 a 2002 para cerca de R\$ 1,05 bilhão em média no período de 2003 a 2013, um aumento de 51,5\% no volume de recursos. A fração correspondente aos recursos do MS, passou de 1,22\% (média de 1995 a 2002) para 1,49\% (média de 2003 a 2013), um aumento de 
0,27 pontos percentuais ou $22 \%$ da fatia inicial. A Fig. 1 mostra as características dessa evolução.

Figura 1 - Recursos federais, em reais deflacionados, alocados à assistência odontológica ambulatorial no SUS nos períodos de 1995 a 2002 e de 2003 a 2013.

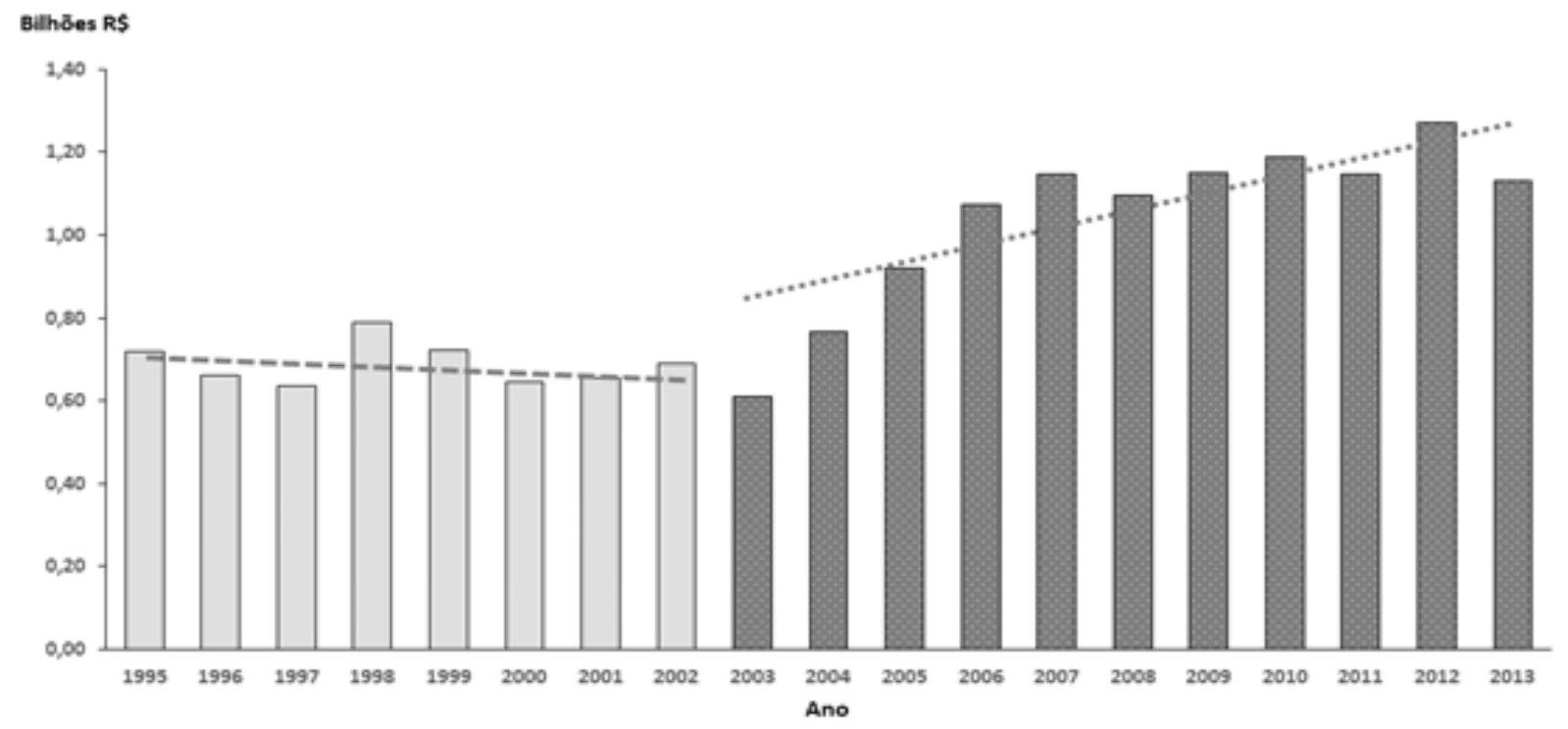

\section{DISCUSSÃO}

Até a realização do presente estudo a literatura sobre a participação do governo federal no financiamento de ações odontológicas ambulatoriais no SUS constituía-se de estimativas e, além disso, circunscrevia-se a períodos curtos de tempo, em geral relativos a um ou outro governo. Era uma limitação importante, que praticamente inviabilizava análises de mais longo prazo. Ademais, não havia referência a algum método capaz de viabilizar tais abordagens. Neste estudo, buscou-se superar as limitações metodológicas para apurar dados relativos especificamente a ações de saúde bucal no âmbito do SUS e, com tais procedimentos, identificar de modo original as principais características do financiamento da área em um período de 18 anos. Os resultados corroboram estudos anteriores $^{(6)(7)}$ que mencionaram aumento na alocação dos recursos à área odontológica, mas divergem quanto à magnitude do aumento e, também, da afirmação de que tal aumento seria contínuo e crescente ${ }^{(7)}$. Desde que os programas de odontologia escolar desenvolvidos pelo Serviço Especial de Saúde Pública (SESP) no início dos anos 1950 marcaram de modo significativo o início da presença do Estado brasileiro nesta área da saúde ${ }^{(12)}$ apenas em 1989 o orçamento da União apresentou, pela primeira vez, dotação específica para ações de saúde bucal, dois anos após a criação da Divisão Nacional de Saúde Bucal na estrutura do $\mathrm{MS}^{(13)}$. Além da não serem especificados rotineiramente, em nível federal, os recursos destinados à área da odontologia pública, inviabilizando o acompanhamento e controle dos custos específicos dessa modalidade assistencial, resta bastante prejudicada qualquer perspectiva avaliativa. Daí a relevância de análises como a empreendida no presente estudo, conforme crítica formulada pelos delegados à $2^{\text {a }}$ Conferência Nacional de Saúde Bucal, realizada em 1993, quanto à necessidade de serem definidos para a Saúde Bucal "financiamento específico" com descentralização das ações, sob controle social, com vistas 
ao desenvolvimento de um novo modelo de atenção em saúde bucal, pois não se constatavam avanços significativos evidenciando-se "baixíssima prioridade" para a área durante os anos 1990(14).

O modo como uma determinada política pública é financiada induz o elenco de ações e operações que a compõe. Assim, para que a PNSB/PBS pudesse adquirir potência para efetivamente reorganizar os serviços odontológicos e ser bem sucedida, impactando o modelo de atenção em saúde bucal, era estratégico que pudesse dispor de mais recursos e induzir à inovação e ruptura com práticas tradicionais, reconhecidamente ineficientes e ineficazes ${ }^{(4)}$. A necessidade de mais recursos decorria do crônico subfinanciamento dessas ações no âmbito do sistema público de saúde, e a ruptura com práticas tradicionais implicava criar mecanismos adequados de transferências capaz de induzir inovações.

Os resultados do presente estudo indicam, porém, que ao contrário do aventado na literatura sobre o tema, foi bastante modesta a porcentagem da ampliação dos recursos federais da saúde alocados à saúde bucal no período de 1995 a 2013, uma vez que este último correspondeu a 1,22\% (1995-2002) e 1,49\% (2003-2013), atingindo o menor valor do período em 1997 (1,09\%) e o maior valor em 2007 (1,72\%), embora se registre um aumento de 51,5\% na média do volume de recursos investidos em saúde bucal de 1995-2002 (R \$ 690 milhões) a 2003-2013 (R \$ 1,05 bilhão). Este resultado diverge do resultado apurado por Kornis et al (2011) $)^{(7)}$ quanto a ter havido um "significativo crescimento do volume de recursos financeiros especificamente voltados para esta área de atenção à saúde”. Ainda que este aumento (de 51,5\%) seja razoável, está muito aquém do cogitado em outros estudos sobre o tema, abrangendo um período histórico próximo ao definido para esta análise, que indicaram aumentos muitas vezes superiores, da ordem de 944,2\%(6) e aproximadamente $1.000 \%$ (como sugerem os gráficos em Nóbrega et al 2010) ${ }^{(5)}$. Além disso, ainda que tenha havido aumento, o volume de recursos alocados à odontologia no âmbito do SUS pelo governo federal está muito aquém das necessidades assistenciais dos brasileiros.

Quanto ao aumento progressivo do gasto público federal com a PNSB/PBS, pode ser admitida apenas em parte, uma vez que houve efetivo aumento em 2003 e 2006, com o volume de recursos públicos federais com ações odontológicas ambulatoriais evoluindo de aproximadamente R\$ 611 milhões para cerca de R\$ 1 bilhão. Nos anos seguintes, porém, de 2007 a 2013 esse volume se estabilizou em torno de R $\$ 1,1$ bilhão. Os resultados do presente estudo não corroboram a afirmação de NOBREGA et al (2010) $)^{(5)}$, de que se observa "um crescente investimento em todas as áreas de complexidade, tanto na saúde em geral como na odontologia".

Cabe assinalar, por fim, a alta complexidade metodológica relacionada com os procedimentos necessários para se apurar os valores que respondem a uma pergunta aparentemente simples: quanto foi gasto com saúde bucal de 1995 a 2013? Conclui-se pela necessidade de desenvolver e aprimorar os mecanismos e sistemas de informações sobre a gestão pública da saúde até que seja possível alcançar um patamar razoável de efetiva transparência em relação a esses gastos. Com as características atuais dos sistemas de informação é praticamente impossível ao cidadão comum, 
aos jornalistas e outros profissionais da comunicação, e mesmo a pesquisadores familiarizados com a avaliação econômica em saúde, acessar esses dados e produzir análises e informações que respondam ao interesse público na saúde da população.

\section{CONCLUSÃO}

Nos termos da análise empreendida pode-se concluir que houve ampliação da ordem de $51,5 \%$ nos recursos alocados à Política Nacional de Saúde Bucal, comparando-se os períodos de 1995 a 2002 (governo Fernando Henrique Cardoso) e 2003 a 2013 (governos Luiz Inácio Lula da Silva e Dilma Rousseff). Tal aumento, porém, não alcançou a magnitude admitida em alguns estudos brasileiros. Essa divergência quanto ao volume de recursos alocados ao financiamento dessa política setorial pode ser atribuída ao fato de que nesses estudos o método adotado implicou operar análises a partir de valores nominais, sem deflacioná-los, enviesando assim os resultados, ademais das dificuldades metodológicas impostas a essas análises pelas características dos sistemas de informações sobre o orçamento público na saúde. Com este estudo busca-se corrigir esta impropriedade metodológica e, aprimorando-se os conhecimentos acerca do tema, disponibilizar dados e informações qualificados e, portanto, mais apropriados para a avaliação econômica em saúde.

\section{REFERÊNCIAS BIBLIOGRÁFICAS}

1. Narvai PC. Avanços e desafios da Política Nacional de Saúde Bucal no Brasil. Tempus Actas Saude Coletiva - Saude Bucal. 2009;21-34.

2. Brasil. Ministério da Saúde. Portaria MS-GM n 2607, de 10 de dezembro de 2004. Aprova o Plano Nacional de Saúde/PNS - Um Pacto pela Saúde no Brasil. Vol. 142, Diário Oficial da União. 2004.

3. Martino LVS. A Política Nacional de Saúde Bucal em municípios da Região Metropolitana de São Paulo , na primeira década do século XXI [Internet]. Universidade de São Paulo; 2011. Available from: http://www.teses.usp.br/teses/disponiveis/6/6135/tde-26012012-112537/pt-br.php

4. Frazão P, Narvai PC. Saúde bucal no SUS: 20 anos de lutas por uma politica pública. Saúde em Debate. 2009;33(81):64-71.

5. Nóbrega CBC, Hoffmann RHS, Pereira AC, Meneghim M de C. Financiamento do setor saúde: uma retrospectiva recente com uma abordagem para a odontologia. Cien Saude Colet [Internet]. 2010;15(suppl 1):1763-72. Available from: http://www.scielo.br/scielo.php?script=sci arttext\&pid=S1413-81232010000700088\&lng=pt\&tlng=pt

6. Pucca Junior GA, Lucena EHG De, Cawahisa PT. Financing national policy on oral health in Brazil in the context of the Unified Health System. Braz Oral Res [Internet]. 2010;24 Suppl 1:26-32. Available from: http://www.scielo.br/scielo.php?script=sci_arttext\&pid=S1806- 
$83242010000500005 \& \operatorname{lng}=$ en\&nrm=iso\&tlng=en

7. Kornis GEM, Maia LS, Fortuna RFP. Evolução do financiamento da atenção à saúde bucal no SUS: Uma análise do processo de reorganização assistêncial frente aos incentivos federais. Physis. 2011;21(1):197-215.

8. Arretche M. Financiamento federal e gestão local de políticas sociais: o difícil equilíbrio entre regulação, responsabilidade e autonomia. Cien Saude Colet. 2003;8(2):331-45.

9. Narvai PC, Frazão P. Avaliação da atenção à saúde bucal. In: Tanaka OY, Ribeiro EL, Almeira CAL de, editors. Avaliação em saúde: contribuições para incorporação no cotidiano. 1st ed. Rio de Janeiro: Atheneu; 2017. p. 185-200.

10. Munhoz D. Economia aplicada: técnicas de pesquisa e análise econômica. Brasília: UnB; 1989.

11. Schwarzer H. Nota sobre efeitos da opção entre diversos índices para deflacionamento de valores nominais. Políticas Sociais Acompan e Análise. 2001;9(1):127-30.

12. Narvai PC. Saúde bucal coletiva: caminhos da odontologia sanitária à bucalidade. Rev Saude Publica. 2006;40(SPEC. ISS.):141-7.

13. Pinto VG. A odontologia brasileira às vésperas do ano 2.000: diagnóstico e caminhos a seguir. Ed.Santos, editor. São Paulo; 1993.

14. Narvai PC, Frazão P. Saúde bucal no Brasil: muito além do céu da boca. Rio de Janeiro: Ed.Fiocruz; 2008. 147 p.

Artigo apresentado em outubro de 2019 Artigo aprovado em janeiro de 2020 Artigo publicado em maio de 2020 